\title{
Enhancement of $T$ cell-mediated and humoral immunity of B-glucuronidase-based DNA vaccines against HPV16 E7 oncoprotein
}

\author{
MICHAL SMAHEL ${ }^{1}$, INGRID POLÁKOVÁ ${ }^{1}$, DANA POKORNÁ ${ }^{1}$, VIERA LUDVÍKOVÁ ${ }^{1}$, \\ MARTINA DUSKOVÁ ${ }^{1}$ and JOSEF VLASÁK ${ }^{2}$ \\ ${ }^{1}$ Department of Experimental Virology, Institute of Hematology and Blood Transfusion, Prague; \\ ${ }^{2}$ Institute of Plant Molecular Biology, Academy of Sciences of the Czech Republic, Ceské Budejovice, Czech Republic
}

Received February 13, 2008; Accepted April 2, 2008

\begin{abstract}
Therapeutic DNA vaccines against oncogenic infection with human papillomavirus type 16 (HPV 16) are mostly targeted against viral oncoproteins E7 and E6. To adapt the E7 oncoprotein for DNA immunization, we have previously reduced its oncogenicity by modification of the Rb-binding site and enhanced immunogenicity of the modified E7GGG gene by the fusion with the 5'-terminus of the gene encoding E. coli $\beta$-glucuronidase (GUS). In this study, we attempted to improve immunogenicity of the GUS-based anti-E7 vaccines by increasing the steady-state level of fusion proteins. We fused deletion mutants of E7GGG and codon-optimized E7GGG with the 5'-terminus of GUS and unaltered E7GGG with the 3'-terminus of GUS. Furthermore, we mutated the initiation codon of the GUS gene in the E7GGG.GUS construct, as GUS alone was produced from this fusion gene. We found that only the fusion of E7GGG with the 3'-terminus of GUS (GUS.E7GGG) and deletion mutants of E7GGG with the 5'-terminus of GUS increased the steady-state level of fusion proteins in transfected human 293T cells. Analysis of immune reactions induced in mice by vaccination via a gene gun showed that the increased steady-state level of fusion proteins resulted in augmented production of E7-specific antibodies, but did not enhance cellmediated anti-tumor immunity. Finally, we joined the signal sequence of the adenoviral E3 protein with GUS.E7GGG. This modification led to the predominant localization of the fusion protein in the endoplasmic reticulum and enhancement of $\mathrm{CD}^{+} \mathrm{T}$-cell response, while antibody production was reduced. In conclusion, we found modifications of the
\end{abstract}

Correspondence to: Dr Michal Smahel, Department of Experimental Virology, Institute of Hematology and Blood Transfusion, U Nemocnice 1, 12820 Prague 2, Czech Republic

E-mail: smahel@uhkt.cz

Key words: DNA vaccine, human papillomavirus, E7 oncogene
E7GGG.GUS fusion gene that augmented either humoral or cell-mediated immune responses.

\section{Introduction}

Vaccination with plasmid DNA is a rapidly developing method for induction of immune responses. It was proved to induce both humoral and cell-mediated immunity, but the level of the immune responses was often unsatisfactory in comparison with traditional vaccination strategies. The efficacy of DNA vaccines can be enhanced by modifications of a gene coding for an antigen. These modifications include changes resulting in the increase of protein production or the alteration of protein stability and/or cellular localization (1).

The E7 oncoprotein of human papillomavirus type 16 (HPV16) is a suitable target for development of therapeutic DNA vaccines against malignant diseases associated with HPV16 infection (2). As it is only a weak immunogen, numerous modifications of the E7 oncogene were constructed and their immunogenicity was examined on mouse tumor models. Some of them have already been tested in clinical trials (3). Enhanced immunity against E7 was demostrated for instance after codon optimization of the E7 sequence (4), addition of intracellular localization signals (5), and fusion with genes encoding chaperones (6), bacterial toxins (7) or viral proteins capable of intercellular spreading (8).

As oncogenicity is a characteristic of the HPV16 E7 gene inconsistent with its use for DNA immunization of humans, we have reduced its tumorigenic potential by the preparation of the modified E7GGG gene encoding the protein with substitution of three amino acids in the pRb-binding site (9). To enhance immunogenicity of E7GGG, we fused it with sorting signals of lysosome-associated membrane protein 1 (LAMP-1) (10), E. coli ß-glucuronidase (GUS) (11), and mouse heat shock protein 70 (Hsp70) (12). As the fusion with GUS (E7GGG.GUS) yielded the highest anti-tumor efficacy, we performed further modifications to enhance its immunogenicity. In this study, we demonstrated that stabilization of the fusion protein increases the generation of E7specific antibodies, but only targeting into the endoplasmic reticulum (ER) enhances cell-mediated immunity and antitumor effect of the DNA vaccine delivered by a gene gun. 


\section{Materials and methods}

Plasmids. The construction of plasmids pBSC/GUS, pBSC/ E7GGG.GUS, and pBSC/E7GGGpGUS (designated pBSC/ E7GGG41.GUS in this report) was described previously (11). To perform some of the modifications specified below, the genes GUS and E7GGG.GUS were cloned into the EcoRI site of pALTER-MAX (Promega, Madison, WI). The modified genes were then cloned back into the EcoRI site of pBSC.

The genes E7GGG60.GUS and E7GGG75.GUS were prepared from the GUS gene by addition of a sequence encoding 60 or 75 amino acids from the $\mathrm{N}$-terminus of the E7GGG protein, respectively. These sequences were amplified from the plasmid pBSC/E7GGG (9) using the forward primer E7-1 (11) and the reverse primer E7-60 (5'-CCAGGATCCC TTGCAACAAAAGGTTACAAT-3') or E7-75 (5'-CCAGGA TCCGTCTACGTGTGTGCTTTGTA-3'), respectively. The PCR products were digested with $\mathrm{Bam} \mathrm{HI}$ and cloned into the BamHI site upstream of the GUS gene.

The termination codon of the GUS gene was eliminated and the HindIII site was created using the Altered Sites II Mammalian Mutagenesis System (Promega) and the primer 5'-CAGGAGAGTTGTTGAAGCTTGTTTGCCTCCCTG-3' (substituted nucleotides are underlined). Into the HindIII site, the E7GGG gene was inserted after amplification with the primers 5'-CACAAGCTTTGATGCATGGAGATACACC TAC-3' (forward) and 5'-CACAAGCTTTTATGGTTT CTGAGAACAGAT-3' (reverse) which resulted in the GUS.E7GGG gene. To prepare the SS.GUS.E7GGG gene containing the signal sequence from the adenoviral E3 gene, annealed oligonucleotides 5'-GATCCGCCGCCATGAGGT ACATGATTTTAGGCTTGCTCGCCCTTGCGGCAGTCT GCAGCGCTGCCG-3' and 5'-GATCCGGCAGCGCTGCAG ACTGCCGCAAGGGCGAGCAAGCCTAAAATCATGTA CCTCATGGCGGCG-3' were cloned into the BamHI site upstream of the GUS.E7GGG gene.

The initiation codon of the GUS gene was eliminated and the XhoI site was created in the resultant EGUS gene using the Altered Sites II Mammalian Mutagenesis System and the primer 5'-TTCTACAGGACGTAACTCGAGGGACT GACCACC-3' (substituted nucleotides are underlined). This modification was also performed in the E7GGG.GUS gene (E7GGG.EGUS).

The E7GGG gene optimized for expression in human cells (hE7GGG; the GenBank accession no. EU443245) was designed and synthesized by GeneArt (Regensburg, Germany). The sequence was amplified using the primers 5'-TTCCGGA TCCATCATGCATGG-3' (forward) and 5'-CATGCATGGG ATTAGGATCC-3' (reverse) and cloned into the BamHI site upstream of the GUS gene (hE7GGG.GUS).

The modified constructs were verified by sequencing. pBSC-derived plasmids were propagated in E. coli, XL1-blue strain, and purified with the Qiagen Plasmid Maxi Kit (Qiagen, Hilden, Germany).

Mice. Six- to eight-week-old female C57BL/6 mice (H-2 ${ }^{\mathrm{b}}$ ) (Charles River, Germany) were used in immunization experiments. Animals were maintained under standard conditions at the Center for Experimental Biomodels, Charles University, Prague.
Cell lines. TC-1 cells prepared by transformation of C57BL/6 primary mouse lung cells with the HPV16 E6/E7 oncogenes and activated H-ras (13) were kindly provided by T.-C. Wu. TC-1/A9 cells with down-regulated MHC class I expression were derived from TC-1 cells that formed a tumor in the mouse immunized against the HPV16 E7 antigen (10). 293 T cells derived from human embryonic kidney cells (14) were kindly provided by J. Kleinschmidt, DKFZ, Heidelberg, Germany. NIH 3 T3 fibroblasts established from mouse embryo culture (15) were obtained from the German National Resource Centre for Biological Material. TC-1, TC-1/A9, and 293T cells were grown in high glucose Dulbecco's modified Eagle's medium (DMEM; PAA Laboratories, Linz, Austria) supplemented with $10 \%$ fetal calf serum (FCS; PAA), $2 \mathrm{mM}$ L-glutamine, $100 \mathrm{U} / \mathrm{ml}$ penicillin, and $100 \mu \mathrm{g} / \mathrm{ml}$ streptomycin. NIH 3T3 cells were cultured in high glucose DMEM supplemented with $10 \%$ calf serum (PAA), 2 mM L-glutamine, $100 \mathrm{U} / \mathrm{ml}$ penicillin, and $100 \mu \mathrm{g} / \mathrm{ml}$ streptomycin.

Analysis of GUS activity. The enzymatic activity of GUS was determined after transfection of $293 \mathrm{~T}$ cells with corresponding plasmids as described previously (11). In brief, cells were transfected by modified calcium-phosphate precipitation in HEPES-buffered saline solution (16), incubated for 2 days, and lysed in the GUS buffer (50 mM phosphate buffer, $\mathrm{pH} 7.0$, $1 \mathrm{mM}$ EDTA, $10 \mathrm{mM}$ ß-mercaptoethanol, $0.1 \%$ Triton X-100). GUS activity was assayed with the 4-methylumbelliferyl-ßD-glucuronide (MUG) substrate. Protein concentration was measured according to Bradford (17).

Immunoblotting staining. $293 \mathrm{~T}$ cells seeded in 6-cm dishes were transfected with $6 \mu \mathrm{g}$ of plasmids by calcium-phosphate precipitation in HEPES-buffered saline solution. Two days after transfection, the cells were washed twice with ice-cold PBS and lysed in the GUS buffer or Laemmli buffer. Proteins separated by SDS-PAGE were electroblotted onto a PVDF membrane (Amersham Biosciences, Little Chalfont, UK), incubated with polyclonal anti-GUS antibodies (Molecular Probes, Eugene, OR) and, subsequently, with secondary peroxidase-labeled antibodies (Amersham Biosciences). Blots were stained using the ECL Plus system (Amersham Biosciences).

Pulse-chase analysis of protein stability. 293T cells grown in $15-\mathrm{cm}$ dishes were transfected with $30 \mu \mathrm{g}$ of plasmids by calcium-phosphate precipitation in HEPES-buffered saline solution. One day after transfection, $2 \times 10^{6}$ cells were seeded in 6-cm dishes. The next day, the cells were starved in methionine/cysteine-free DMEM supplemented with 5\% dialyzed FCS (PAA) for $1 \mathrm{~h}$ and incubated with ${ }^{35} \mathrm{~S}$-labeled methionine/cysteine $(100 \mu \mathrm{Ci} / \mathrm{ml}$ in DMEM with $5 \%$ dialyzed FCS; GE Healthcare). After washing with PBS, the cells were cultured in DMEM supplemented with $1 \mathrm{mM}$ methionine and $0.5 \mathrm{mM}$ cysteine for indicated time intervals and lysed in the RIPA buffer with a protease inhibitor cocktail (Sigma, Saint Louis, MO) added. The E7-containing fusion proteins were immunoprecipitated using anti-E7 monoclonal antibody (clone TVG710Y; Santa Cruz Biotechnology, Santa Cruz, CA) and protein G PLUS-Agarose (Santa Cruz Biotechnology) and separated by SDS-PAGE. The dried gels were exposed to 
the Fuji BAS-MS imaging plate and luminescence was measured by the Fuji FLA 2000 phosphorimager (Fujifilm, Stamford, CT) and analyzed with ScanPack 3.0 software (Biometra, Goettingen, Germany).

Immunofluorescence staining. NIH 3 T3 cells were grown on coverslips in 24-well plates and transfected with $0.5 \mu \mathrm{g}$ plasmids using the Metafectene transfection reagent (Biontex, Martinsried/Planegg, Germany). Two days after transfection, cells were fixed in methanol for $10 \mathrm{~min}$ at $-20^{\circ} \mathrm{C}$. The E7 antigen was stained with E7-specific mouse monoclonal antibody (clone 8C9; Zymed, San Francisco, CA) followed by Alexa Fluor 488-conjugated goat anti-mouse IgG antibodies (Molecular Probes). Simultaneously, the ER or the Golgi apparatus (GA) were stained with rabbit anti-calreticulin or anti-GM130 antibodies (both Santa Cruz Biotechnology), respectively, and secondary Alexa Fluor 546-conjugated goat anti-rabbit IgG antibodies (Molecular Probes). The slides were examined by a Leica TCS SP2 AOBS confocal microscope, images were processed by Leica Confocal Software (Leica Microsystems, Germany).

Preparation of gene gun cartridges. Plasmid DNA was coated onto 1- $\mu$ m gold particles (Bio-Rad, Hercules, CA) as described previously (9). Each cartridge contained $0.5 \mathrm{mg}$ of gold particles coated with $1 \mu \mathrm{g}$ DNA.

Immunization experiments. Mice were immunized with plasmids by a gene gun (Bio-Rad) at a discharge pressure of 400 psi into the shaven skin of the abdomen. Each immunization consisted of one shot delivering $1 \mu \mathrm{g}$ of plasmid DNA. In immunization/challenge experiments, mice were vaccinated with two doses administered at a 2 -week interval, and 2 weeks after the last vaccination the animals were challenged s.c. into the back with $3 \times 10^{4}$ TC-1/A9 cells suspended in $150 \mu 1 \mathrm{PBS}$. In therapeutic immunization experiments, mice were first inoculated with $3 \times 10^{4}$ TC-1 cells and immunized 3 and 10 days later. Tumor cells were administered under anesthesia with intraperitoneal etomidate (0.5 mg/mouse; Janssen Pharmaceutica, Beerse, Belgium). Tumor growth was monitored twice a week. Tumor size was calculated from three perpendicular measurements using the formula:

$$
\frac{\pi}{6}(\mathrm{a} \times \mathrm{b} \times \mathrm{c})
$$

ELISPOT assay. Mice were immunized by a gene gun with two doses of plasmids given at a two-week interval. Two weeks after the second dose, an ELISPOT assay was performed as described previously (11). Cells producing interferon $\gamma(\mathrm{IFN}-\gamma)$ were detected in lymphocyte bulk cultures prepared from splenocytes of three immunized animals and cultivated with

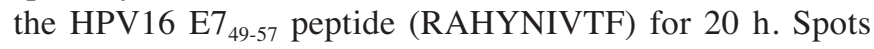
were evaluated by the Eli.Scan ELISPOT Scanner (A.EL.VIS, Hannover, Germany).

Intracellular cytokine staining (ICS). Lymphocytes were prepared and cultivated with the E749-57 peptide as for an ELISPOT assay. Twelve hours before staining, GolgiStop (BD
Biosciences Pharmingen, San Diego, CA) was added to the culture medium according to the manufacturer's instructions. Surface CD8 molecules were stained with PE-conjugated rat anti-mouse CD8a monoclonal antibody (BD Biosciences Pharmingen). Then, cells were fixed and permeabilized using the Cytofix/Cytoperm kit (BD Biosciences Pharmingen) and intracellular IFN- $\gamma$ was stained with FITC-conjugated rat anti-mouse IFN- $\gamma$ monoclonal antibody (BD Biosciences Pharmingen). The stained cells were measured on a Coulter Epics XL flow cytometer (Coulter, Miami, FL) and analyzed by FlowJo 7.1.2. software (TreeStar, Ashland, OR).

ELISA. Sera were collected from mice immunized for the ELISPOT assay and ICS. E7-specific antibodies were detected in sera diluted 1:50 by ELISA as described previously (12).

Statistical analysis. Tumor formation in the immunization experiments was analyzed by a log-rank test. Tumor growth was evaluated by two-way analysis of variance. Results were considered significantly different at $\mathrm{P}<0.05$. Calculations were performed using GraphPad Prism 4.0 software (GraphPad Software, San Diego, CA).

\section{Results}

Generation of deletion mutants of E7GGG.GUS and 3'terminus fusion GUS.E7GGG and characterization of their steady-state level production. At least in some conditions, cross-presentation of antigens seems to be the principal mechanism of induction of immune responses after DNA immunization with a gene gun (18). Therefore, we tried to enhance the efficacy of the E7GGG.GUS vaccine by modifications of the fusion gene that could increase the steadystate level of resultant proteins. In our previous study, we showed that while fusion of the full-length E7GGG gene with GUS decreases the steady-state level of the GUS antigen about 10-fold, expression of the fusion gene E7GGG41.GUS containing 41 amino acids from the N-terminus of E7GGG is comparable with that of GUS alone (11). As E7GGG41.GUS does not contain the H-2 ${ }^{\text {b }}$ RAHYNIVTF epitope (aa 49-57), we constructed other two deletion mutants, E7GGG60.GUS and E7GGG75.GUS, comprising 60 and 75 amino acids from the E7GGG N-terminus, respectively (Fig. 1).

Stability and/or immunogenicity of fusion proteins can be influenced by position of fusion partners. This effect has also been demonstrated for the HPV16 E7 oncogene fused with the mouse Hsp70 gene (19). To verify the position effect of the E7GGG fusion with GUS, we joined E7GGG with the 3 '-terminus of GUS, thus generating the GUS.E7GGG gene (Fig. 1).

We have demonstrated previously that fusion of E7GGG with GUS does not markedly affect the enzymatic activity of GUS (11). Therefore, we measured the GUS activity to quantitatively compare the production of fusion proteins after transfection of 293T cells with pBSC-derived plasmids (Fig. 2A). We found that the steady-state level of the 5'terminus fusion proteins decreased with increasing the portion of E7GGG added to GUS. The main difference (corresponding to about $60 \%$ of the enzymatic activity of GUS alone) was found between the production of the proteins E7GGG60.GUS 


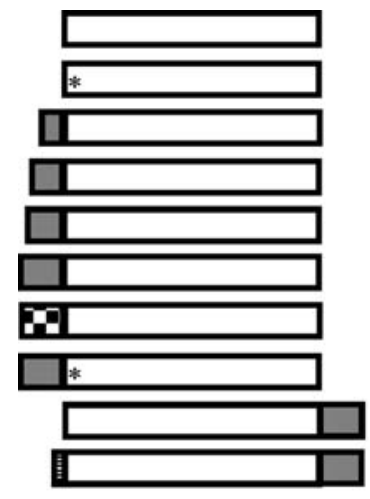

GUS

EGUS

E7GGG41.GUS

E7GGG60.GUS

E7GGG75.GUS

E7GGG.GUS

hE7GGG.GUS

E7GGG.EGUS

GUS.E7GGG

SS.GUS.E7GGG

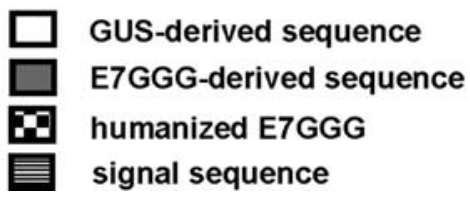

Figure 1. Schematic representation of genes cloned into the mammalian expression plasmid pBSC. An asterisk $\left(^{*}\right)$ indicates the mutation in the GUS initiation codon.

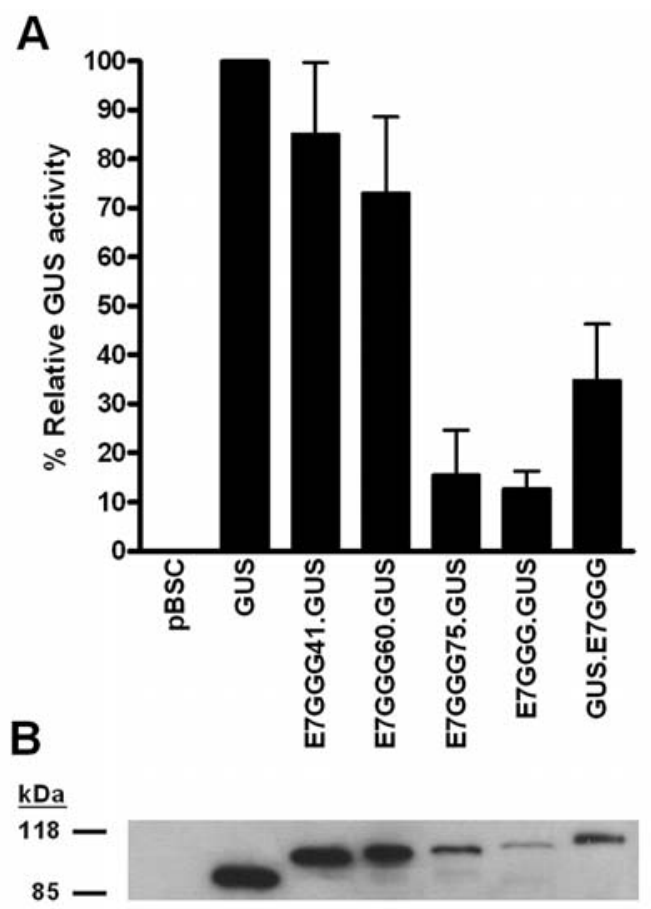

Figure 2. Expression of deletion mutants and C-terminus fusion. 293T cells were transfected with pBSC-derived plasmids, lysed in GUS buffer two days thereafter, and tested for the production of proteins: A, Analysis of GUS activity by incubation with the MUG substrate - columns, mean of three independent experiments; bars \pm SD. B, Immunoblotting staining with antiGUS antibodies. Two $\mu \mathrm{g}$ of proteins were separated in $7 \%$ SDS-PAGE gel.

and E7GGG75.GUS. The GUS activity of GUS.E7GGG was about twice higher than that of E7GGG.GUS. These quantitative data were confirmed by immunoblotting analysis of cell lysates (Fig. 2B).

Immunogenicity of deletion mutants and 3'-terminus fusion. We examined immunogenicity of GUS fusion genes after
DNA immunization of C57BL/6 mice by a gene gun. In our experimental setting, induction of E7-specific antibodies by E7GGG.GUS was negligible. All modified genes elicited higher production of antibodies against E7. The level of antibodies correlated with the steady-state level of protein production in transfected 293T cells (Fig. 3A).

Cell-mediated immunity against E7 was tested by an ELISPOT assay detecting splenocytes that produced IFN- $\gamma$ after restimulation with the $\mathrm{H}-2 \mathrm{D}^{\mathrm{b}} \mathrm{E} 7_{49-57}$ epitope. The number of IFN- $\gamma$-producing cells after immunization with E7GGG.GUS was at least twice higher than after immunization with the modified fusion genes (Fig. 3B). Furthermore, we examined the anti-tumor effect of the modified genes. For induction of s.c. tumors, we used TC- 1 cells producing the E7 oncoprotein and their derivative with the reduced surface expression of MHC class I molecules, TC-1/A9 cells. In preventive immunization against TC-1/A9 cells, all mice developed a tumor with the exception of two animals immunized with the E7GGG.GUS gene (Fig. 3C), which corresponds with the highest efficiency of this gene in the ELISPOT assay. In therapeutic immunization against TC-1 cells, both the E7GGG.GUS gene and the modified genes induced elimination of tumor cells in about half of mice (Fig. 3D). In summary, the increase in the steady-state level of the GUS fusion proteins enhanced the production of E7-specific antibodies, but did not improve the cytotoxic T-lymphocyte (CTL) response.

Subsequent modifications of E7GGG fusions with GUS: optimization of E7GGG, elimination of the GUS initiation codon and addition of a signal sequence. Codon optimization can substantially increase the production of a protein. Such modification of the HPV16 E7 gene resulted in enhanced immune responses after DNA immunization $(4,20)$. This effect was achieved even after fusion of the codon optimized E7 gene with the unmodified lysosome-associated membrane protein 1 (LAMP-1) (21). Therefore, we joined the E7GGG gene optimized for expression in human cells (hE7GGG) with GUS, thus generating the hE7GGG.GUS gene. However, despite the fact that the hE7GGG gene produced about a 6-fold higher amount of the E7GGG protein in comparison with the E7GGG gene (data not shown), production of the E7GGG.GUS protein from the hE7GGG.GUS and E7GGG.GUS genes was comparable (Fig. 4A).

After detection of the E7GGG.GUS protein with anti-GUS polyclonal antibody, we found a minor band corresponding in size with the GUS protein. We supposed that the translation of the E7GGG.GUS gene could occasionally start from the GUS initiation codon. To test this hypothesis and possibly increase the production of the E7GGG.GUS fusion protein, we mutated the GUS initiation codon, which resulted in the substitution of methionine for glutamic acid (E7GGG.EGUS). Similarly, we introduced this mutation into the GUS gene (EGUS). By using the GUS-specific antibodies, immunoblotting analysis of lysates from the transfected 293T cells showed that the mutation eliminated the production of the protein corresponding in size with the GUS protein from both of the mutated genes (Fig. 4B). However, the steady-state level of the mutated E7GGG.EGUS protein was a bit lower than that of the E7GGG.GUS protein. Moreover, the GUS 
A

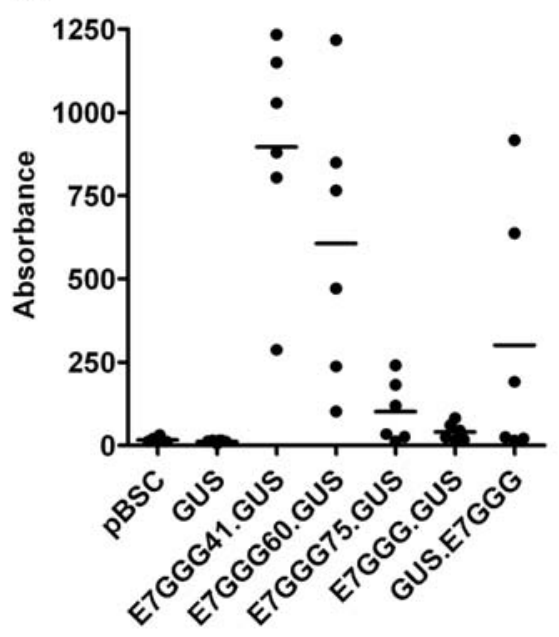

C

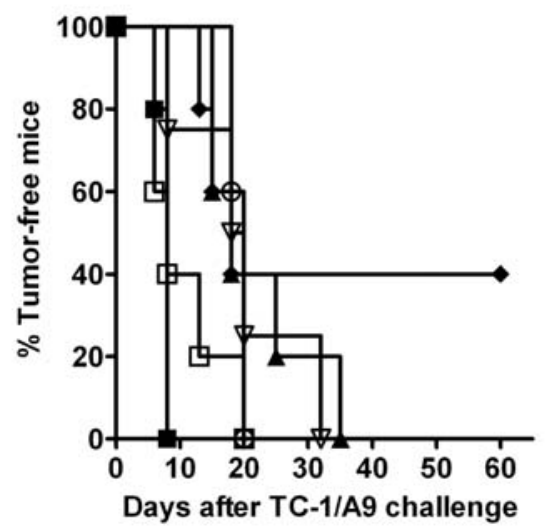

B

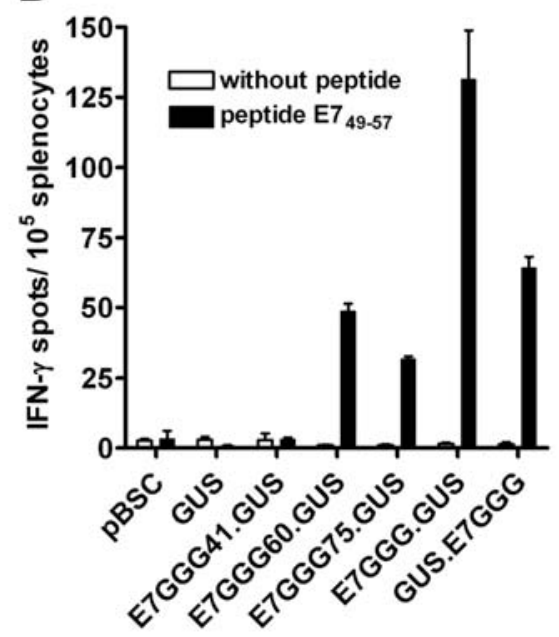

D

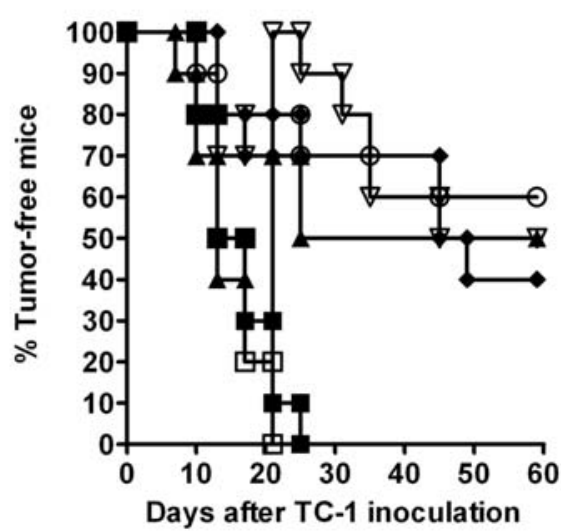

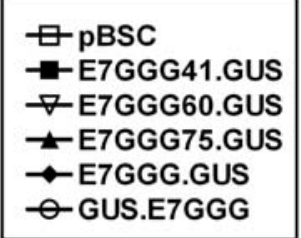

Figure 3. Immunogenicity of deletion mutants and C-terminus fusion. A and B, Mice ( $\mathrm{n}=3$ ) were twice immunized at a 2 -week interval by a gene gun with $1 \mu \mathrm{g}$ of plasmids and two weeks after the second immunization, blood was collected and lymphocyte bulk cultures were prepared from splenocytes. A, E7-specific antibodies were examined in 1:50 diluted sera by ELISA. B, Lymphocytes producing IFN- $\gamma$ were detected after incubation with the peptide RAHYNIVTF by an ELISPOT assay. Control lymphocytes were cultivated without the peptide. Columns, mean of duplicate samples; bars \pm SD. C, Mice ( $=5$ ) were twice immunized at a 2-week interval by a gene gun and challenged s.c. with $3 \times 10^{4}$ TC-1/A9 tumor cells two weeks later. D, Mice ( $\mathrm{n}=10$ ) were inoculated s.c. with $3 \times 10^{4}$ TC- 1 cells and immunized by a gene gun 3 and 10 days later.

A

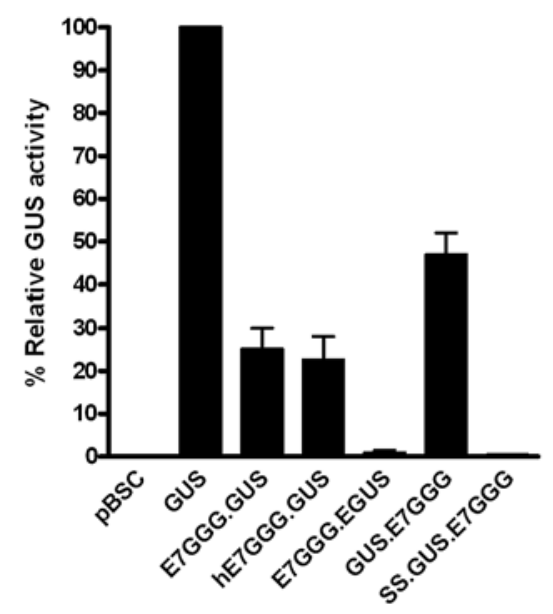

B

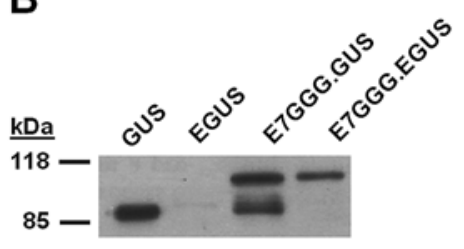

C

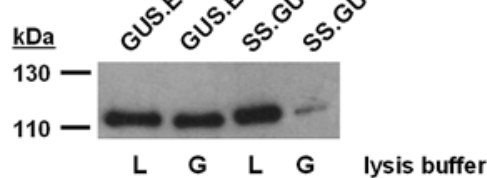

D

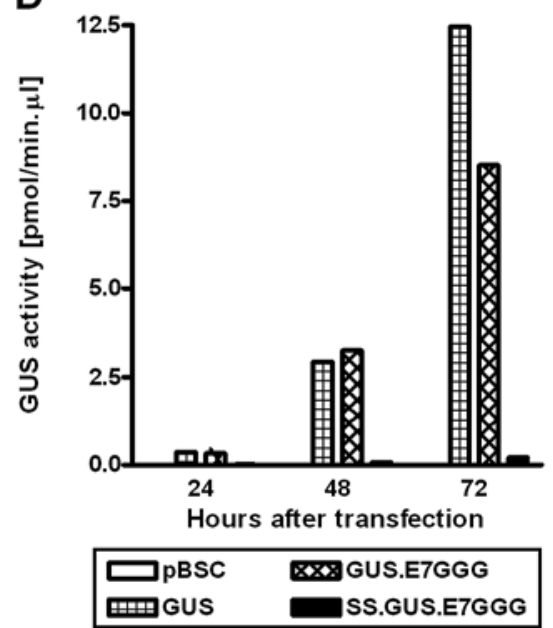

Figure 4. Expression of the modified genes hE7GGG.GUS, E7GGG.EGUS, and SS.GUS.E7GGG. The production of the fusion proteins was examined after transfection of $293 \mathrm{~T}$ cells with pBSC-derived plasmids. A and B, Two days after transfection, cells were lysed in the GUS buffer and GUS was detected in lysates by analysis of GUS activity with the MUG substrate (A, columns, mean of three independent experiments; bars \pm SD) or by immunoblotting staining with antiGUS antibodies [B, $4 \mu \mathrm{g}$ (GUS) or $13 \mu \mathrm{g}$ of proteins (EGUS, E7GGG.GUS, E7GGG.EGUS) were separated in 7\% SDS-PAGE gel]. C, Two days after transfection, cells were lysed in the GUS (G) or Laemmli buffer (L) and proteins in $2 \mu 1$ of lysates were separated in $7 \%$ SDS-PAGE gel, transferred onto a PVDF membrane and stained with anti-GUS antibodies. D, Samples of media were collected 24, 48, and $72 \mathrm{~h}$ after transfection and examined for GUS activity. 
A
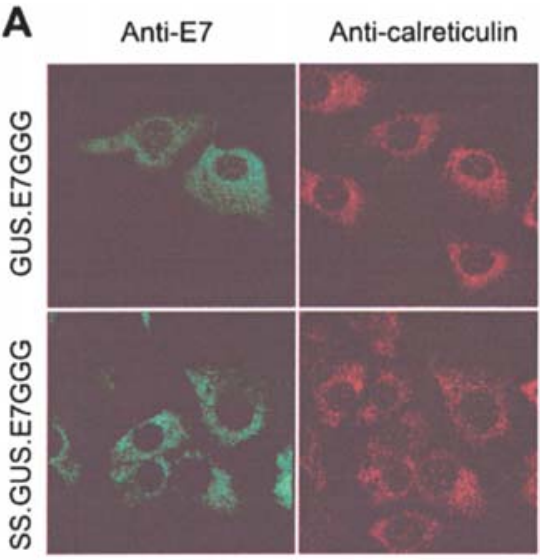

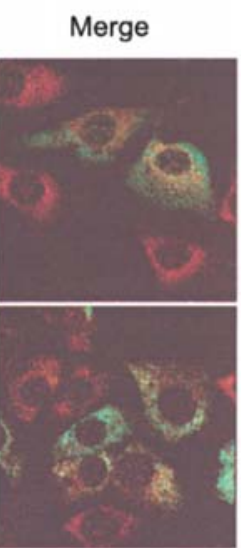

B Anti-E7

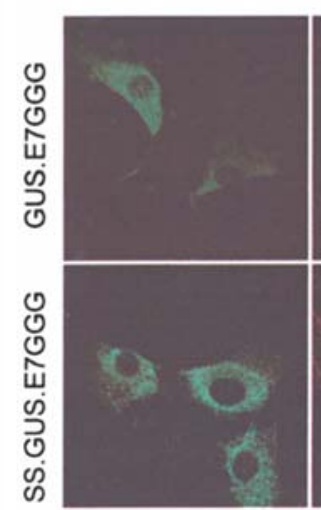

Anti-GM130

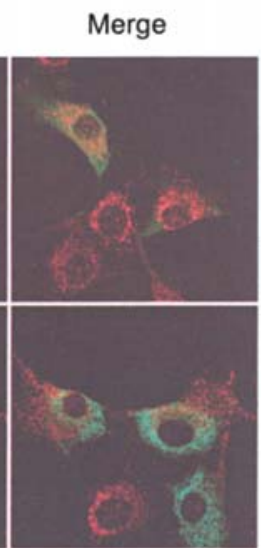

Figure 5. Intracellular localization of SS.GUS.E7GGG. NIH 3 T3 cells were transfected with plasmids pBSC/GUS.E7GGG or pBSC/SS.GUS.E7GGG and fixed with methanol 2 days thereafter. The E7 antigen was stained using anti-E7 monoclonal antibody (green). Simultaneously, the ER (A) or GA (B) were stained using polyclonal anti-calreticulin or anti-GM130 antibodies, respectively (red). The slides were examined by a confocal microscope.

activity of E7GGG.EGUS was almost completely eliminated (Fig. 4A). These data suggest that the enzymatic activity of the mutated protein was reduced by the modification of its conformation caused by the substitution of methionine for glutamic acid.

The efficacy of DNA vaccines can be enhanced by the alteration of antigen cellular localization. As addition of a signal sequence targeting a protein into the ER has been reported to increase immunogenicity of some cytoplasmic or nuclear proteins including the $\mathrm{E} 7$ protein $(5,22)$, we joined the adenoviral E3 signal sequence with GUS.E7GGG (SS.GUS.E7GGG). This modification resulted in almost complete elimination of the GUS activity in lysates of the transfected 293T cells (Fig. 4A). Immunoblotting analysis showed that while after lysis with the Laemmli buffer the level of the detected SS.GUS.E7GGG protein was comparable with that of the GUS.E7GGG protein, it was markedly reduced in the GUS lysis buffer (Fig. 4C), which suggested localization of the SS.GUS.E7GGG protein into subcellular compartments. Furthermore, we tested possible secretion of the SS.GUS.E7GGG protein by determination of the GUS activity in media of the transfected cells. However, the enzymatic activity of SS.GUS.E7GGG was about 40-fold lower when compared with GUS.E7GGG (Fig. 4D). The higher amount of GUS.E7GGG in the medium was confirmed by immunoblotting analysis (data not shown), which excluded the possibility that a post-translational modification caused the lower GUS activity of the SS.GUS.E7GGG protein. Furthermore, colocalization analysis demonstrated that while the E7-specific antibody diffusely stained the cytoplasm of the NIH 3T3 cells transfected with the GUS.E7GGG gene, the SS.GUS.E7GGG protein showed perinuclear staining and colocalization with calreticulin, a chaperone resident in the ER, but not with GM130, a marker of the GA (Fig. 5).

Immunogenicity of hE7GGG.GUS, E7GGG.EGUS, and SS.GUS.E7GGG. Similarly to E7GGG.GUS, the modified genes hE7GGG.GUS, E7GGG.EGUS, and SS.GUS.E7GGG elicited a negligible production of E7-specific antibodies (data not shown). Examination of cell-mediated immunity by intracellular staining of IFN- $\gamma$ in $\mathrm{CD}^{+} \mathrm{T}$ lymphocytes showed that only immunization with SS.GUS.E7GGG
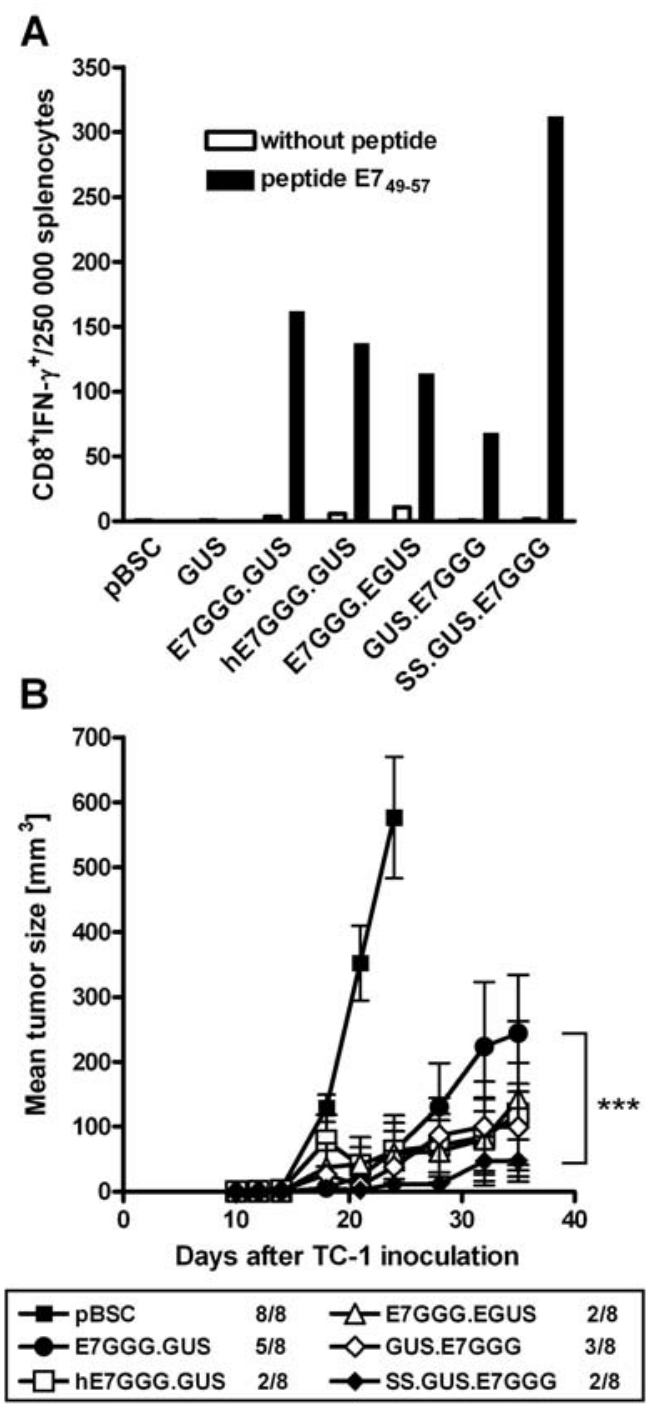

Figure 6. Immunogenicity of the genes hE7GGG.GUS, E7GGG.EGUS, and SS.GUS.E7GGG. A, Mice $(n=3)$ were twice immunized at a 2 -week interval by a gene gun with $1 \mu \mathrm{g}$ of plasmids and two weeks after the second immunization, lymphocyte bulk cultures were prepared from splenocytes. $\mathrm{CD}^{+}$lymphocytes producing IFN- $\gamma$ were detected after incubation with the peptide RAHYNIVTF by ICS. Control lymphocytes were cultivated without the peptide. B, Mice $(n=8)$ were inoculated s.c. with $3 \times 10^{4}$ TC-1 cells and immunized by a gene gun 3 and 10 days later. No. of mice with a tumor/no. of mice in group is indicated. 
A

\section{E7GGG.GUS E7GGG60.GUS E7GGG.EGUS}

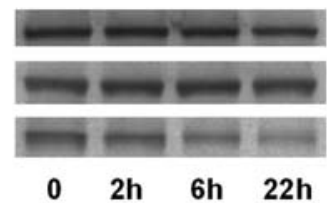

B

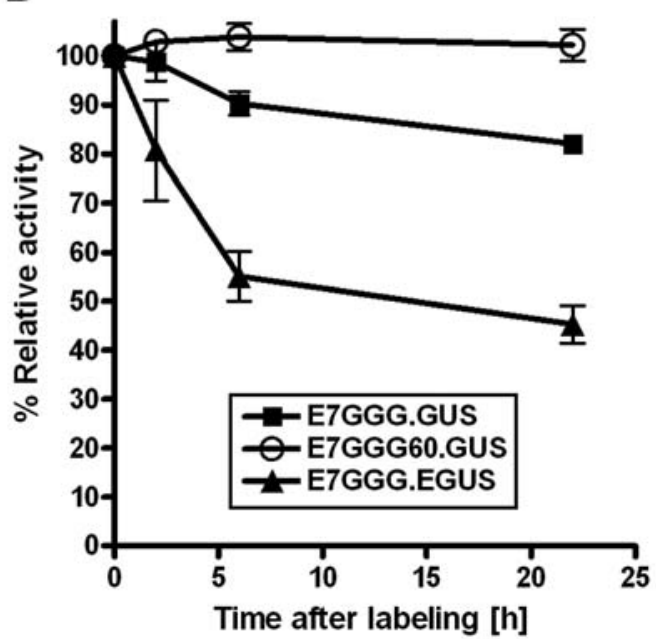

Figure 7. Stability of proteins E7GGG.GUS, E7GGG60.GUS, and E7GGG.EGUS. 293T cells were transfected with pBSC-derived plasmids, starved in a methionine/cysteine-free medium for $1 \mathrm{~h}$, and incubated with ${ }^{35} \mathrm{~S}$-labeled methionine/cysteine for $1 \mathrm{~h}$. After cultivation in the medium containing methionine/cysteine for indicated time-points, the cells were lysed and the E7-containing fusion proteins were immunoprecipitated using anti-E7 monoclonal antibody and analyzed by SDS-PAGE and phosphorimaging (A). Protein quantification was performed using ScanPack 3 software. The data represent means of two independent experiments; bars $\pm \mathrm{SD}(\mathrm{B})$

enhanced this immune response in comparison with the original E7GGG.GUS gene (Fig. 6A). Accordingly, after therapeutic vaccination against TC- 1 cells, the growth of tumors in mice immunized with SS.GUS.E7GGG was significantly slower $(\mathrm{P}<0.001)$ compared with E7GGG.GUSimmunized animals (Fig. 6B). In summary, localization of the fusion protein of E7GGG with GUS into the ER increased the CTL response, but did not affect the induction of antibodies against E7.

Fusion protein stability. Immunogenicity of DNA vaccines can be substantially influenced by the stability of the produced immunogens $(23,24)$. As modifications of the E7GGG.GUS gene resulted in considerable differences in the steady-state level of the produced fusion proteins possibly caused by the alteration of protein stability, we compared the stability of the cytoplasmic proteins E7GGG.GUS, E7GGG60.GUS, and E7GGG.EGUS by pulse-chase labeling followed by the immunoprecipitation with anti-E7 monoclonal antibody. We found that the stability of these proteins corresponded with their steady-state level in transfected 293T cells (Fig. 7). While the amount of the labeled E7GGG60.GUS protein did not recognizably decrease after 22-h chase, about a half of E7GGG.EGUS and 20\% of E7GGG.GUS was degraded. Our data suggest that the stability of fusion proteins corresponded with the induction of E7-specific antibodies but we did not find any relation between the protein stability and cell-mediated immune responses.

\section{Discussion}

The rate of antigen synthesis is an important factor determining the efficacy of DNA vaccines. It can be affected by both the plasmid backbone and the sequence of the gene encoding the antigen (25). Moreover, the steady-state level of an antigen in transfected cells can be increased by the stabilization of the antigen, which might enhance its cross-presentation. As cross-priming was identified as the predominant mechanism inducing a cytotoxic T-lymphocyte (CTL) response after DNA immunization by a gene gun (18), we attempted to enhance the steady-state level of the HPV16 E7 antigen produced after DNA vaccination. We showed previously that the fusion of the E7 protein modified in the pRb-binding site (E7GGG) with the N-terminus of GUS (E7GGG.GUS) increased the steady-state level of the E7 antigen about 20-fold and substantially enhanced the efficacy of gene-gun DNA immunization (11). To increase further the steady-state level of the E7 antigen, here we modified the E7GGG.GUS fusion gene by: i) the deletions of E7GGG portions encoding Cterminal amino acids, ii) the fusion of E7GGG with the 3' GUS terminus (GUS.E7GGG), iii) the codon optimization of E7GGG, and iv) the elimination of the GUS initiation codon. However, only deletion mutants and the GUS.E7GGG fusion led to increase in the E7 production. Moreover, none of the modifications improved the CTL response when compared with E7GGG.GUS, despite the fact that the augmented steady-state level of fusion proteins corresponded with the improvement of production of E7-specific antibodies, which suggested that a higher amount of the E7 antigen was released from transfected cells and thus E7 cross-priming might also be enhanced.

Codon optimization of E7GGG (hE7GGG) increased the E7GGG protein production about 6 -fold, but we did not record any effect after the fusion of the codon-optimized E7GGG with GUS - both the production of the fusion protein and immune responses induced by gene-gun immunization were comparable with those of the E7GGG.GUS gene. We suppose that the translation of the non-optimized GUS part of the hE7GGG.GUS gene (that is about 6-fold longer than the hE7GGG part) suppressed the beneficial effect of E7GGG optimization.

The HPV16 E7 antigen is an unstable protein with a halflife of about $30-60 \mathrm{~min}(26,27)$. We have shown previously that the steady-state level of the E7 antigen can be substantially augmented by fusion of E7GGG with the N-terminus of GUS (11), but induction of E7-specific antibodies was still negligible in our experimental setting. Only after further increase of the E7 antigen level achieved by the removal of E7GGG C-terminal amino acids from the E7GGG.GUS protein (i.e. in constructs E7GGG41.GUS, E7GGG60.GUS, and E7GGG75.GUS) or by fusion of E7GGG with the C-terminus of GUS, we elicited production of anti-E7 antibodies that corresponded with the steady-state level of the E7 antigen and the stability of the fusion proteins in transfected cells as demonstrated for E7GGG60.GUS. Antibodies against 
E7 do not probably contribute to the protection against HPVassociated tumors, but stabilization of an antigen by fusion with GUS could be utilized for the induction of antibodies against other infectious agents or preparation of poly- or monoclonal antibodies.

Stability of the E7 antigen also affects induction of cellmediated immunity. For instance, the destabilization of the E7 antigen by the mutations in zinc-binding motifs (23) or the fusion with ubiquitin $(28,29)$ significantly enhanced CTL responses. We prepared modifications of the E7GGG.GUS protein that are either more (E7GGG60.GUS) or less stable (E7GGG.EGUS) than the original protein. However, none of them induced a stronger CTL response. Our results are in accordance with the data reported by Golovina et al (30) who showed that while targeted degradation of an antigen substantially enhanced epitope production, misfolding of an antigen caused by a point mutation or deletion, did not impact on epitope production despite decreasing antigen stability.

Intracellular and extracellular targeting of antigens is another key factor influencing immunogenicity of DNA vaccines and modulating immune responses $(1,25)$. Addition of a signal sequence targeting an antigen into the ER and possibly into a secretory pathway can enhance humoral and/or cell-mediated immune responses after DNA immunization. However, both cellular localization and the type of enhanced immune response after fusion with a signal sequence depend on the nature of the antigen $(31,32)$. Addition of a signal sequence to the E7 oncoprotein probably resulted in E7 secretion and an improved E7-specific CTL response $(5,22)$. Therefore, in the SS.GUS.E7GGG construct, we fused the signal sequence from the adenoviral E3 gene to the GUS.E7GGG gene. We showed that the protein produced from the SS.GUS.E7GGG gene accumulated in the ER and was not secreted from cells. In comparison with the E7GGG.GUS gene, SS.GUS.E7GGG did not enhance the production of E7-specific antibodies, but improved the anti-tumor effect mediated by $\mathrm{CD}^{+} \mathrm{T}$ lymphocytes. We suppose that the retrograde transport of the fusion protein from the ER into cytosol followed by degradation with proteasomes (32) could be responsible for the enhanced immunogenicity of SS.GUS.E7GGG.

In conclusion, we observed that the increase in the steadystate level of the GUS-based fusion proteins containing HPV16 E7-derived sequences augmented the production of antibodies against E7, but did not enhance CTL responses. Localization of the fusion protein into the ER had an opposite effect. These results further evoke the issue of the contribution of cross-priming versus direct priming to the induction of anti-tumor immunity with GUS-based DNA vaccines.

\section{Acknowledgements}

We thank V. Navratilova, P. Vesela, and K. Riegerova for technical help and O. Sebesta for assistance with confocal microscopy. This work was supported by grant. no. 521/05/ 2092 from the Czech Science Foundation and grant. no. NR9246-3/2007 from the Ministry of Health of the Czech Republic and by the League against Cancer, Prague. The doctoral study of I. Poláková was supported by grant. no. 310/05/H533 from the Czech Science Foundation.

\section{References}

1. Leifert JA, Rodriguez-Carreno MP, Rodriguez F and Whitton JL: Targeting plasmid-encoded proteins to the antigen presentation pathways. Immunol Rev 199: 40-53, 2004.

2. zur Hausen H: Papillomaviruses and cancer: from basic studies to clinical application. Nat Rev Cancer 2: 342-350, 2002.

3. Hung CF, Monie A, Alvarez RD and Wu TC: DNA vaccines for cervical cancer: from bench to bedside. Exp Mol Med 39: 679-689, 2007.

4. Liu WJ, Gao F, Zhao KN, Zhao W, Fernando GJ, Thomas R and Frazer IH: Codon modified human papillomavirus type 16 E7 DNA vaccine enhances cytotoxic T-lymphocyte induction and anti-tumour activity. Virology 301: 43-52, 2002.

5. Ji H, Wang TL, Chen CH, Pai SI, Hung CF, Lin KY, Kurman RJ, Pardoll DM and Wu TC: Targeting human papillomavirus type $16 \mathrm{E} 7$ to the endosomal/lysosomal compartment enhances the antitumor immunity of DNA vaccines against murine human papillomavirus type 16 E7-expressing tumors. Hum Gene Ther 10: 2727-2740, 1999.

6. Chen $\mathrm{CH}$, Wang TL, Hung CF, Yang Y, Young RA, Pardoll DM and $\mathrm{Wu}$ TC: Enhancement of DNA vaccine potency by linkage of antigen gene to an HSP70 gene. Cancer Res 60: 1035-1042, 2000.

7. Hung CF, Cheng WF, Hsu KF, Chai CY, He L, Ling M and Wu TC: Cancer immunotherapy using a DNA vaccine encoding the translocation domain of a bacterial toxin linked to a tumor antigen. Cancer Res 61: 3698-3703, 2001.

8. Hung CF, Cheng WF, Chai CY, Hsu KF, He L, Ling M and Wu TC: Improving vaccine potency through intercellular spreading and enhanced MHC class I presentation of antigen. J Immunol 166: 5733-5740, 2001.

9. Smahel M, Sima P, Ludvikova V and Vonka V: Modified HPV16 E7 genes as DNA vaccine against E7-containing oncogenic cells. Virology 281: 231-238, 2001.

10. Smahel M, Sima P, Ludvikova V, Marinov I, Pokorna D and Vonka V: Immunisation with modified HPV16 E7 genes against mouse oncogenic TC-1 cell sublines with downregulated expression of MHC class I molecules. Vaccine 21: 1125-1136, 2003.

11. Smahel M, Pokorna D, Mackova J and Vlasak J: Enhancement of immunogenicity of HPV16 E7 oncogene by fusion with $E$. coli beta-glucuronidase. J Gene Med 6: 1092-1101, 2004.

12. Pokorna D, Mackova J, Duskova M, Rittich S, Ludvikova V and Smahel M: Combined immunization with fusion genes of mutated E7 gene of human papillomavirus type 16 did not enhance antitumor effect. J Gene Med 7: 696-707, 2005.

13. Lin KY, Guarnieri FG, Staveley OK, Levitsky HI, August JT, Pardoll DM and Wu TC: Treatment of established tumors with a novel vaccine that enhances major histocompatibility class II presentation of tumor antigen. Cancer Res 56: 21-26, 1996.

14. DuBridge RB, Tang P, Hsia HC, Leong PM, Miller JH and Calos MP: Analysis of mutation in human cells by using an Epstein-Barr virus shuttle system. Mol Cell Biol 7: 379-387, 1987.

15. Jainchill JL, Aaronson SA and Todaro GJ: Murine sarcoma and leukemia viruses: assay using clonal lines of contact-inhibited mouse cells. J Virol 4: 549-553, 1969.

16. Kingston RE, Chen CA, Okayama H and Rose JK: Calcium phosphate transfection. In: Current Protocols in Molecular Biology. Ausubel FA, Brent R, Kingston RE, et al (eds.) John Wiley \& Sons, New York, pp9.1.4-9.1.11, 1997.

17. Bradford MM: A rapid and sensitive method for the quantitation of microgram quantities of protein utilizing the principle of protein-dye binding. Anal Biochem 72: 248-254, 1976.

18. Cho JH, Youn JW and Sung YC: Cross-priming as a predominant mechanism for inducing CD8(+) T cell responses in gene gun DNA immunization. J Immunol 167: 5549-5557, 2001

19. Li Y, Subjeck J, Yang G, Repasky E and Wang XY: Generation of anti-tumor immunity using mammalian heat shock protein 70 DNA vaccines for cancer immunotherapy. Vaccine 24: 5360-5370, 2006

20. Cid-Arregui A, Juarez V and zur Hausen H: A synthetic E7 gene of human papillomavirus type 16 that yields enhanced expression of the protein in mammalian cells and is useful for DNA immunization studies. J Virol 77: 4928-4937, 2003.

21. Kim MS and Sin JI: Both antigen optimization and lysosomal targeting are required for enhanced anti-tumour protective immunity in a human papillomavirus E7-expressing animal tumour model. Immunology 116: 255-266, 2005. 
22. Michel N, Osen W, Gissmann L, Schumacher TN, Zentgraf H and Muller M: Enhanced immunogenicity of HPV 16 E7 fusion proteins in DNA vaccination. Virology 294: 47-59, 2002.

23. Shi W, Bu P, Liu J, Polack A, Fisher S and Qiao L: Human papillomavirus type 16 E7 DNA vaccine: mutation in the open reading frame of E7 enhances specific cytotoxic T-lymphocyte induction and antitumor activity. J Virol 73: 7877-7881, 1999.

24. Bins AD, Wolkers MC, van den Boom MD, Haanen JB and Schumacher TN: In vivo antigen stability affects DNA vaccine immunogenicity. J Immunol 179: 2126-2133, 2007.

25. Manoj S, Babiuk LA and van Drunen Littel-van den Hurk S: Approaches to enhance the efficacy of DNA vaccines. Crit Rev Clin Lab Sci 41: 1-39, 2004.

26. Smotkin D and Wettstein FO: The major human papillomavirus protein in cervical cancers is a cytoplasmic phosphoprotein. $\mathrm{J}$ Virol 61: 1686-1689, 1987.

27. Reinstein E, Scheffner M, Oren M, Ciechanover A and Schwartz A: Degradation of the E7 human papillomavirus oncoprotein by the ubiquitin-proteasome system: targeting via ubiquitination of the N-terminal residue. Oncogene 19: 5944-5950, 2000.
28. Liu WJ, Zhao KN, Gao FG, Leggatt GR, Fernando GJ and Frazer IH: Polynucleotide viral vaccines: codon optimisation and ubiquitin conjugation enhances prophylactic and therapeutic efficacy. Vaccine 20: 862-869, 2001.

29. Leachman SA, Shylankevich M, Slade MD, Levine D, Sundaram RK, Xiao W, Bryan M, Zelterman D, Tiegelaar RE and Brandsma JL: Ubiquitin-fused and/or multiple early genes from cottontail rabbit papillomavirus as DNA vaccines. J Virol 76: 7616-7624, 2002.

30. Golovina TN, Morrison SE and Eisenlohr LC: The impact of misfolding versus targeted degradation on the efficiency of the MHC class I-restricted antigen processing. J Immunol 174: 2763-2769, 2005.

31. Rice J, King CA, Spellerberg MB, Fairweather $\mathrm{N}$ and Stevenson FK: Manipulation of pathogen-derived genes to influence antigen presentation via DNA vaccines. Vaccine 17: 3030-3038, 1999.

32. Golovina TN, Wherry EJ, Bullock TN and Eisenlohr LC: Efficient and qualitatively distinct MHC class I-restricted presentation of antigen targeted to the endoplasmic reticulum. J Immunol 168: 2667-2675, 2002. 